\title{
Influencia de la variable tiempo en el uso de las tecnologías de la información y comunicación (TIC) en clases por profesores: el caso de la Universidad de La Guajira, Colombia
}

\author{
Marlin A. Aarón ${ }^{1,2}$ y Ernesto F. Bautista ${ }^{3}$ \\ (1) Facultad de Ingeniería, Universidad de La Guajira Ciudadela Universitaria Km 5 Riohacha La Guajira, Colombia, \\ (2) Grupo de investigación y desarrollo Motivar (correo-e: maaron@uniguajira.edu.co) \\ (3) Universidad Internacional Iberoamericana de México UNINI Calle 15 núm. 36, entre 10 y 12, IMI III Campeche, \\ México. CP 24560 (correo-e: ernesto.bautista@unini.edu.mx)
}

Recibido May. 15, 2021; Aceptado Jul. 9, 2021; Versión final Ago. 18, 2021, Publicado Dic. 2021

\begin{abstract}
Resumen
La presente investigación examina la influencia de la variable tiempo en el uso de las tecnologías de la información y la comunicación (TIC) por parte del profesorado de la Universidad de la Guajira (Colombia). El enfoque es cuantitativo (método ex-post-facto) y cualitativo (descriptivo-interpretativo), con una encuesta respondida por 380 profesores. Los resultados muestran que el $59.38 \%$ de los profesores considera que es altamente útil usar las TIC. Las franjas del día en la que más usan las TIC son en la mañana y en la tarde (28.31\%). El más alto porcentaje (33.68\%) de profesores que usa las TIC en clases casi siempre miden el tiempo, de una hora a dos horas (36.75\%). Un porcentaje $(32.55 \%)$ un poco menor expresa que no lo tiene medido. Se concluye que la percepción positiva de los profesores hacia las TIC es alta y que una mínima proporción de profesores no la usan.
\end{abstract}

Palabras clave: TIC; tiempo de clases; educación superior; competencia del profesorado

\section{Impact of the time variable on in-class use of information and communication technologies (ICT) by professors: the case of the University of La Guajira, Colombia}

\begin{abstract}
This research study examines the influence of the time variable on how professors use information and communication technologies (ICT) in-class at the University of La Guajira (Colombia). The research approach is quantitative (ex-post-facto method) and qualitative (descriptive-interpretative). A total of 380 professors are surveyed. The results show that $59.38 \%$ of the professors surveyed consider that ICTs are highly useful. The time of the day that professors most use ICT are mornings and afternoons (28.31\%). The highest proportion $(33.68 \%)$ of professors almost always measure the amount of time they use ICTs in-class, which ranges between one and two hours (36.75\%), but a lower percentage (32.55\%) do not measure time. It is concluded that professors demonstrate a highly positive perception towards ICTs and that there is a small percentage of professors that do not used them.
\end{abstract}




\section{INTRODUCCIÓN}

El uso de las TIC dentro de una Institución educativa demanda esfuerzos enormes (Silvia y Astudillo, 2013). Esto no se aparta de las Instituciones de educación superior, donde la enseñanza de disciplinas y saberes requiere que el ejercicio de la docencia se haga a tono con las exigencias que la sociedad tiene sobre ese profesional que está formando. En este siglo XXI, la sociedad demanda que las instituciones estén en armonía con sus avances y que el andamiaje tecnológico está presente en todas las actividades formativas que se proponen en el aula de clases. De manera global instituciones educativas y sociedad generan acciones para que unas y otras estén conectadas a partir de la tecnología. Por ejemplo, los estudiantes se matriculan en las instituciones usando dispositivos tecnológicos y la sociedad sobre esta acción de matriculación, reclama y genera información que es acumulada, accedida, procesada y divulgada usando también tecnología. Se usa tecnología que se produce en la sociedad industrial, que se pasea por la sociedad educativa, que genera información en todos los ámbitos y escalas y se devuelve para generar más exigencias sobre cómo mejorar la forma en que esta tecnología es producida y en esto las Instituciones educativas aportan.

Las aulas de las Instituciones educativas en las que participan estudiantes y profesores, no pueden estar ausentes del uso de las tecnologías. En este caso nos referimos a las TIC. Los profesores no solo deben ser expertos en los saberes disciplinares, sino también en el uso de TIC, acorde a las exigencias que la sociedad hace. Pero, en algunas instituciones por razones de infraestructura civil, de servicios de energía, de dominio tecnológico u otras, las exigencias globales no se perciben. A pesar que actualmente existen políticas públicas e institucionales orientadas al fortalecimiento de la incorporación de tecnologías en las instituciones de educación en todos los niveles, la fusión educación y TIC se ve distante, inclusive no se perciben como mediaciones para la formación del profesional que egresa a la sociedad a generar trasformaciones a partir de sus saberes y que no cuenta con las habilidades que esta demanda en la que el acceso a información, análisis y categorización está básicamente soportado por el uso de tecnologías. Este profesional que se forma en las instituciones educativas, desde que ingresa debe estar permeado por el uso de las TIC en su tránsito por el currículo con el cual se propende por la formación. Es necesario que estos currículos estén pródigamente mediados por el uso de estas tecnologías para que las competencias digitales y tecnológicas que estos estudiantes adquieran, se mantengan siempre actualizadas. Esto hace necesario que el profesor que en la institución es el mediador entre este estudiante y su acceso a contenidos, apropiación de conocimiento, habilidades y competencias, cuente también con las digitales, no solo para su uso desde lo disciplinar, sino para enseñar. Debe hacer uso de estas TIC y convertirlas en apoyo a su saber y también a su hacer.

Por ello, las instituciones de educación superior deben poner en marcha proyectos para la educación permanente y continua de su equipo de profesores que incluye en la actualidad, el uso de TIC. La forma como usan los profesores las TIC, (Morrissey, 2008), podría estar sometida o afectada por factores que pueden no ser tan evidentes y no se tienen en cuenta en la planificación de estos proyectos. Identificar la forma como son usadas las TIC por ellos teniendo en cuenta la influencia del tiempo, es el problema científico que aquí se plantea. Para ello se analiza la influencia del tiempo, el tiempo de uso de las TIC, el tiempo asignado y el tiempo realmente usado por los profesores, el tiempo secuencial o disruptivo para uso de TIC, el momento de la clase en que las usa, el tiempo social, la productividad de este tiempo, a fin de que se puedan encontrar relaciones, diferencias, razones o pretextos, que ayuden a proponer una argumentación sobre la influencia que tiene en el "cómo" usan las TIC los profesores en las Instituciones educativas universitarias, en este caso en la Universidad de La Guajira. Al poner en marcha proyectos tecnológicos institucionales es posible que la influencia de la utilización del tiempo genere resultados distintos a lo planificado y esto se convierta en un problema. Un problema que debe ser investigado.

Dentro de los propósitos de estos proyectos, la institución educativa puede estar poniendo en marcha una política de estado para aportar a la disminución de brechas digitales, apoyando la enseñanza o mejorando su infraestructura tecnológica y si esto no fue logrado, a pesar de que el proyecto fue lógica y estructuralmente planificado, es un problema que posibilita y demanda una investigación. El proyecto tecnológico pudo ser planeado usando técnicas para ello, pero al momento de ser ejecutado, los logros o resultados esperados se redujeron o no se alcanzaron. Los contextos hacen diferencia frente a la forma como se ponen en marcha los proyectos en las instituciones educativas universitarias. Es posible que un proyecto que se desarrolló excelentemente en una institución, no ocurra lo mismo en otras al pretender extenderlo y las razones de esto, podrían estar influenciadas por la forma como utilizaron el tiempo dedicado a las TIC o sus percepciones. Las preguntas de investigación que se identifican son, ¿Cuál es la influencia del tiempo en el uso que hacen los profesores de la Universidad de La Guajira al uso de las TIC? ¿Los profesores tienen una percepción sobre el tiempo de uso de las TIC en clases? El objetivo de este artículo es analizar la influencia de la variable tiempo en la forma como usa las TIC el profesorado en la Universidad de la Guajira. La investigación que soporta este artículo tiene un ámbito más amplio y estudia no solo el impacto del tiempo sino el del espacio en que se usan las TIC en clases por profesores universitarios. Se gestó desde la necesidad de reconocer el sentido de estos aspectos y su impacto en la estructuración de proyectos tecnológicos en el ámbito universitario para su puesta en marcha. 


\section{OTROS ANTECEDENTES}

El tiempo es una variable que hace presencia en muchos aspectos asociado a la vida y aún más allá de ella. Su concepto es complejo y es identificado desde varias disciplinas y saberes de muchas formas, pero todas ellas lo colocan en un lugar trascendental frente al hacer cotidiano, la ciencia e incluso desde la mitología. En esta, se reconocen a tres personajes asociados al tiempo, Cronos (Kronos), Aión y Cairos (Kairós). El primero, el dios Cronos, hijo de Urano el Cielo y de Gea, la Tierra. Fue maldecido por Urano, a quien Cronos castró y a su vez fue derrotado por Zeus, su propio hijo. Es reconocido como regidor del cosmos y simbólicamente asociado al dominio del tiempo y la vida de los humanos no se escapa a ello. Es el dios del tiempo secuencial; es el dios de lo plano, de la agenda, de la cronología y los objetivos. Aión, es representado usando dos figuras, un anciano, señor del tiempo y de lo inmutable, eterno, perfecto y como un joven que sostiene un zodíaco por el que circulan estaciones, reflejando con ello el movimiento cíclico que simboliza la perfección del movimiento y la vinculación de todas las cosas entre sí. Es dios de la eternidad al que no le hace falta devorar nada para ser eterno. Es a la vez niño y anciano. Es el dios de la acción. Aión se ha mostrado como el dios no originado, un dios que no nace y que tampoco muere. Kairós hijo de Zeus, nieto de Cronos y de la diosa de la suerte, Tijé, se identifica como un duende. Kairós representa la oportunidad, el momento oportuno, en el tiempo oportuno que se ofrece al hombre, que hay que saber aprovechar. En imágenes y grafías aparece como un joven con un mechón de cabello, que tiene de la mano la imagen de una balanza desequilibrada (Cladellas, 2009; Ham, 2013).

El tiempo está presente en todo, así como su medición y establecimiento. Desde el reloj solar, el calendario lunar, recorriendo por las dinastías egipcias y los calendarios asociados a las estaciones, las horas temporales, las horas del equinoccio, relojes de agua, la noche, el día, las estrellas, el zodíaco, el astrolabio, las campanas y la hora pública, el péndulo, el año, los meses y los días hasta los modernos relojes de hoy, que están conectados a mecanismos muy sofisticados como el Smartphone (Lucas, 2018). La precisión de la medición del tiempo, está asociada al "para que" de esa medición. El tiempo es una medida fundamental de la física y su medida en el sistema Internacional de unidades es el segundo, sin que exista un objeto que la represente, cómo si existen para otras medidas. La cronometría es la parte de la física que se ocupa de esta medida exacta del tiempo. Esta dimensión del tiempo, hace parte del estudio de esta investigación.

En el documento, "La percepción del tiempo: una revisión desde la neurociencia cognitiva", Correa et al., (2006), presentan una revisión a varios conceptos sobre el tiempo. Indican que, desde la filosofía, Aristóteles creía en la existencia de un tiempo absoluto. El tiempo se consideraba como un marco de referencia fijo, inmutable, sobre el que van sucediendo los acontecimientos. De este modo, era lógico pensar que el tiempo había existido desde siempre. Los mismos autores, expresan que Kant, opinaba lo contrario, "el tiempo es únicamente una condición subjetiva de nuestra intuición humana, (que es siempre sensible, es decir, en la medida en que somos afectados por objetos) y en sí mismo, fuera del sujeto, no es nada". Además, "el tiempo es la forma de la intuición de nosotros mismos y de nuestro estado interior. El tiempo no puede ser una determinación de los fenómenos externos, no pertenece ni a la figura ni a la situación, sino que determina la relación de las representaciones en nuestros estados internos." (ibídem, 2006). Presentan el tiempo cómo una flecha, que siempre cambia; que va de atrás hacia adelante y que se puede ver desde tres (3) flechas, la termodinámica o la dirección del tiempo en la que el desorden aumenta; la cosmológica, la dirección del tiempo en la que el universo está expandiéndose y la flecha psicológica que corresponde a la dirección en la que se siente que pasa el tiempo; la dirección en la que se recuerda el pasado, pero no el futuro. Al decir de los autores, tomando como referente a Kant, la psicología tiene mucho que ver con el tiempo, al considerarlo como "un fenómeno resultante de nuestra forma de percibir el mundo".

Newton en Principia (Chandrasekhar, 2003) describe al tiempo como absoluto, verdadero y matemático, sin relación a algo exterior, discurre uniformemente y se llama duración. El tiempo relativo, aparente y vulgar, es esa medida sensible y externa de una parte de duración cualquiera (igual o desigual), tomada del movimiento: tales son las medidas de horas, de días, de meses, etc., que se usan ordinariamente en lugar del tiempo verdadero. [...] Puede ser que no exista movimiento regular (completamente periódico) por el cual el tiempo pueda ser exactamente medido. Todos los movimientos pueden ser acelerados y retardados. Pero el flujo del tiempo absoluto no puede ser cambiado. La duración o existencia persistente de las cosas es siempre la misma, ya sean los movimientos rápidos o nulos.

Einstein aborda el sentido de la «relativización» del concepto de tiempo: cada sistema de referencia posee necesariamente su propio tiempo. Así, observadores que se muevan con velocidades diferentes ordenarán de manera diferente entre ellos los mismos acontecimientos: lo que un observador ve como simultáneo, para otro puede ocurrir según una diferente secuencia temporal. Por ello dos sucesos que ocurren en lugares distintos del universo no pueden nunca definirse como «simultáneos» en un sentido absoluto, (Malquori, 2011). Cladellas (2009) recogiendo los conceptos históricos del tiempo, lo presenta como cíclico y lineal, significaciones que aún existen en la realidad; la primera en el calendario y la segunda en el cronómetro e 
indica que la concepción como avance lineal sin repetición cíclica es la dominante hoy en día. El mismo autor Cladella (2009), presenta el tiempo asociado al concepto financiero, "el tiempo es oro" frase con la que pretende otorgarle al tiempo una consideración "invaluable" que está por encima de lo que se puede contar.

Correa et al., (2006) han estudiado la percepción del tiempo desde la psicología experimental e indican que es imposible imaginar un organismo cuyas interacciones con el ambiente no queden enmarcadas en las coordenadas espaciotemporales por las que se rigen los acontecimientos naturales. Por tanto, la evolución de un sistema que sea sensible a la dimensión temporal parece un requisito básico para la supervivencia tanto en los sucesos externos como en los internos. Wittmann y van Wassenhove, (2009) teniendo en cuenta postulados de Block et al., (1997) estudian la forma como el ser humano percibe o reconoce el tiempo, indicando que se pueden identificar relojes internos en el ser humano que se encargan de medir situaciones que pasan en el acontecer. Señalan que el "sentido del tiempo" es peculiar y además que el tiempo es intangible. No se puede señalar con el dedo un 'objeto de duración' como se puede hacer con cualquier otro objeto físico, pero es posible experimentar el paso del tiempo cuando uno espera que algo suceda o termine. Entre estos relojes internos se identifica un reloj similar a un cronómetro, un marcapasos que produce una serie de pulsos y el número de pulsos registrados durante un período de tiempo dado, representa la duración subjetiva. Otro reloj es el circadiano, sintonizado para medir duraciones en torno a las horas del día; regula los ritmos diarios en aspectos fundamentales de fisiología y comportamiento. Está formado por neuronas situadas en el hipotálamo que se encargan de seguir y aportar al control de los horarios de vigilia, sueño, de alimentación, entre otros. Se parte de la noción de que el tiempo es una construcción mental y que el cerebro debe derivar activamente estimaciones del tiempo a partir de múltiples fuentes de información, tanto fuera como dentro de los límites del yo mental y material. En la medida en que la experiencia del tiempo está ligada al estado mental del espectador, refleja el estado cognitivo y el bienestar emocional de uno y esta medida del tiempo, lo indican como el reloj cognitivo.

Ferreira y Esteves, (2016) indican que las características personales y los elementos del contexto pueden generar una percepción del tiempo diferente del real y desigual en personas que se encuentran en un mismo lugar. Para comprender esto se deben valorar las condiciones que rodean a las personas, su cultura, el entorno laboral al que pertenece, sus características como sexo y formación, los roles que cumple, entre otras. Otra forma de reconocer las diferentes percepciones que se tienen del tiempo, está dado a través de la forma metafórica como nos referimos a él. La forma como lo hacemos generalmente está dada desde una combinación espacio temporal, que indica movilidad. Hablamos de tiempo atrás, o el martes viene después del lunes, o septiembre viene después de agosto. Hay una sensación espacio temporal que se concibe de manera automática indicando hacía adelante o hacía atrás o en ese momento. La percepción del tiempo es una de las dimensiones que es objeto de estudio en esta investigación.

Es viable medir el tiempo utilizando la periodización. Es una parte de la construcción de la historia que ayuda a estructurar su narración de manera ordenada, (Blanch y Fernández, 2010). Comprender la periodización también es una forma de dominio o de control sobre el tiempo histórico. La cronología que ayuda a medir el tiempo, se activa cómo un mecanismo de control en el que la ocurrencia de los eventos se asocia al tiempo en que ocurre. Los calendarios han sido formas de controlar los ritmos de la vida de las personas, desde las sociedades agrícolas hasta las industriales. "La cronología corresponde a la representación de un tiempo objetivo, que necesitamos para situar los acontecimientos históricos, así como para ordenarlos en una sucesión o, en algunos casos, para decidir la simultaneidad de los hechos. Sin embargo, la cronología no nos dice nada sobre la calidad o el valor de lo que se mide. La cronología no es el tiempo histórico. Es un requisito para aprenderlo pues sitúa los cambios y las continuidades, pero no los explica". Blanch y Fernández (2010). Esta dimensión del tiempo, hace parte del estudio de esta investigación.

Penetrando en el espacio donde se desarrolla este estudio que es el aula de clases, es necesario activar nuestra atención sobre el tiempo y lo que ocurre con él en el aula, a cargo del profesor. La gestión del tiempo en el aula de clases, es motivo de atención, organización y planificación. Martinic y Villalta, (2015) a tono con la obra y reflexión de Husti, (1992) y Roth et al., (2008), consideran que el tiempo es un recurso que deberá ser gestionado con distinto grado de flexibilidad según los objetivos, métodos pedagógicos y necesidades de aprendizaje de los estudiantes y esto es una acción directa que debe emprender el profesor. El tiempo de duración de la clase, el ritmo de aprendizaje que está asociado al tiempo, el momento en que hacer esto o aquello en al aula, el tiempo de inicio y el tiempo final, así como varios aspectos más, son escenarios que han sido estudiados y siguen siéndolo por la gran importancia que tienen para los procesos de enseñanza y aprendizaje. Hjalmarsson (2019), nos invita a relacionar el tiempo directamente con el rendimiento académico de los estudiantes y esto es identificado como un indicador de calidad para medir aspectos que tienen que ver con la forma como funciona el aula y su organización.

El profesor es el líder del ambiente de enseñanza aprendizaje (Hunzicker, 2017). Acorde a esto, el profesor cuenta con un liderazgo distribuido en el que le corresponde definir un ambiente para que la enseñanza se 
logre y se alcance el aprendizaje por parte de sus estudiantes. Para ello, organiza lo pedagógico, lo ambiental no solo desde las relaciones con sus directivos y compañeros, sino también al interior del aula, acorde a la misión institucional y a su productividad. El aula, sea física o virtual, es esencial para realizar la actividad profesoral.

El profesor como líder del proceso define la puesta en marcha de una metodología desde lo pedagógico, condiciona el ambiente del aula y diseña un escenario que sea propicio para la construcción de conocimiento, individual, colectivo y colaborativo, lo que se organiza y planifica acorde a las consideraciones institucionales, curriculares y atendiendo el desarrollo de procesos y productos. Este diseño tiene en cuenta elementos que entre los que podemos listar aspectos de la estructura civil como tamaño, ubicación, iluminación, acústica y otros como entorno con la naturaleza, conectividad, recursos tecnológicos y mobiliario, todo para propiciar un ambiente académico idóneo, Gil-Madrona y otros (2012). En cuanto a la estructura y organización del ambiente del aula, hay un elemento que también debe tenerse en cuenta y es el de mantener un bajo nivel de obsolescencia en el tiempo. Esto es, debe ser sensible a que su estructura física, aprendizajes, enseñanzas, recursos y lo que ocurra dentro de ella, no se envejezcan rápidamente y responda a las exigencias vigentes de la sociedad.

El trabajo del profesor tiene una dimensión fundamental y es la forma como construye o interpreta el tiempo, no solo él, sino también sus compañeros y la institución. El tiempo para el profesor, es un horizonte subjetivo definido que lo posibilita o limita. Lafleur (1999) tomando como contribución lo expresado por Hargreaves, (1992) que indica además que en lo que se refiere al trabajo del profesor tiene cinco dimensiones, "el tiempo técnico-racional, el tiempo fenomenológico, el tiempo físico, el tiempo sociopolítico, el tiempo micro político", registra que estos tiempos se encuentran interrelacionados entre sí. Al reexaminar Lafleur el significado de los tiempos del profesorado presentados por Hargreaves, quien registra al tiempo técnico-racional como una condición instrumental de organización que puede ser manipulada desde la institución con el fin de impulsar la mejora de los cambios educativos cuyo propósito y grado de importancia han sido determinados. Al tiempo micro político le corresponde la distribución del tiempo; los horarios de los diferentes profesores, el horario de cursos y asignaturas cómo algo más que una guía objetiva para saber quién está, dónde y a qué hora. El tiempo fenomenológico, es una dimensión en la cual el tiempo es subjetivo, es vivido, tiene una duración que varía de persona a persona. Pasa de distinta forma para el profesor y para los estudiantes centrado en la vivencia de cada uno. El tiempo físico, sustentado en el principio de Einstein, universalmente aceptado, de que el tiempo físico es relativo. No hay puntos fijos en el espacio o en el tiempo. En cuanto al tiempo sociopolítico, este hace referencia a la forma como se le organiza el tiempo al profesor por parte de la institución.

En estos cinco (5) tiempos acordes a sus fines, habrán de darse resultados o productos por parte del profesor, enseñanza, investigación, organización planeación, proposiciones, entre otros, como parte de su accionar y esto es parte de una de las dimensiones que es objeto de estudio en este estudio, la productividad que genera en su tiempo de clases, con uso de TIC. Una productividad que se da en un tiempo en el que hay relaciones entre docentes y estudiantes y en el que el uso del tiempo no es individual. No se usa el tiempo en un solitario momento del tiempo. Coexiste en medio de muchos tiempos. "Mi tiempo", "tu tiempo", "nuestro tiempo", son tiempos personales que coexisten en una multiplicidad de tiempos. Un momento personal en el que los tiempos sociales se desarrollan. Se pasa del "yo" al "nosotros" al "otro" y se establece simultáneamente una base temporal colectiva más fuerte que la individual con características diferenciadas que las generalmente permitidas en el contexto antropológico e histórico (Adam, 2013).

De estos tiempos de trabajo del profesor, también conectamos el tiempo fenomenológico que apunta a la relatividad del tiempo, a lo subjetivo que es. La percepción del tiempo es subjetiva, de allí la importancia que tiene la percepción del profesor sobre él, como condición de inteligibilidad para acceder a lo real, Oliva (2020), lo que también está relacionado con las teorías de la física sobre la unidad del espacio-tiempo, formulado inicialmente por Minkowski (Petkov y Minkowski, 2010) y desarrolladas por Einstein (Einstein y Hawking, 2007) y en los últimos años por el físico inglés Stephen Hawking, (Cervantes-Cota et al., 2020).

López et al., (2020), respalda que la universalidad del tiempo afecta a todos en el aula. Todos sabemos del tiempo, lo sentimos, lo vivimos, pero cada uno desde su individualidad y esto hace que el tratamiento científico - especulativo del mismo, sea a desiguales niveles. La combinación del tiempo como variable física, con la forma como cada sujeto lo apropia, motivado por su vivencia, hace que las perspectivas del tiempo dependan de lo que viven en el aula. Y por ello, la vivencia del tiempo es algo complejo. Todo lo que ocurre en el aula, se hace desde la planificación del tiempo. El aprendizaje y la enseñanza están supeditados a él y el tiempo está supeditado a la enseñanza y el aprendizaje. Una relación biunívoca que deja huella en profesores y estudiantes y esta huella, también está asociada a elementos del tiempo, en razón de las transformaciones que se de en ambos, por la acción que ocurre en el tiempo en que comparten el espacio, que es el aula. 
Con respecto al uso de las Tecnologías de la Información y la Comunicación TIC en el aula por los profesores, ellos como agentes de cambio y como profesionales deben asumir el papel de líder que favorezca su incorporación. Shepherd et al., (2020) reafirman que un ingrediente clave para que los educadores utilicen con éxito las tecnologías en sus aulas de manera "generativa" y constructivista, son sus creencias sobre la eficacia de las TIC, de otra forma los profesores son reticentes a cualquier tecnología que no les facilite la consecución de los diversos objetivos que el sistema educativo les marca. Y esta resistencia de los profesores a utilizar TIC puede ser una razón importante del fracaso de la introducción plena de las TIC en las aulas. En el mismo sentido se pronuncia Arancibia et al (2018), indicando que el impacto de la integración de las TIC en el aprendizaje depende de cómo el maestro las maneje, valore y use en sus clases. Por esto, es valiosa la afirmación de Sáez (2015) quien considera que en definitiva, las actitudes de los profesores hacia una metodología efectiva para el uso de las tecnologías, se convierten en un factor esencial para la inclusión de las TIC en los contextos educativos, pues a partir de una concepción positiva de los métodos activos y las ventajas del uso de herramientas versátiles y con beneficios pedagógicos, los profesores llevarán a cabo una labor de formación, dedicación de tiempo y diseño de actividades orientadas en este sentido.

Ancira (2011) reitera que para los profesores la integración de la tecnología a la enseñanza es un proceso complejo para mejorar el aprendizaje, en el que el profesor, las necesidades de acceso y capacitación, la cultura escolar y la percepción del profesor son centrales. Falco (2017), reitera lo expresado por Fullan (2007) en el sentido que la capacitación docente es importante, pero "... si no está vinculada al aprendizaje colectivo, no puede influir a la cultura de la escuela". La concepción del profesor a como vincular las TIC en el aula de clases es uno de los elementos centrales, aunque no es el único. Las concepciones son construcciones mentales de los sujetos que tienen relación con experiencias vividas en su contexto, (Arancibia et al, 2018). Esta concepción del profesor sobre usar las TIC en clases se convierte en una de las variables objeto de estudio en esta investigación.

En las revisiones bibliográficas previas (Arancibia et al, 2018) (Falco, 2017), (Sáez, 2015), (Shepherd et al., 2020), se observa que la actitud del profesor, su dominio en el uso de las TIC y la implementación de una pedagogía en contexto, se convierten en elementos que posibilitan de manera exitosa la forma como se puedan incorporar estas en el aula de clases. Autores como Bauer y Kenton (2005) citados por Tammaro y D'Alessio (2016), consideran que cuando el profesor se inicia en el uso de las TIC en el aula de clases, no solo se da una innovación tecnológica, también se da pedagógica, porque generar una fusión, una integración, se consideran modificaciones en los diseños de clases que implican una doble innovación. Una u otra, son grandes posibilitadoras o anuladoras de la integración de las TIC en el aula. Es importante tener en cuenta que estas integraciones se convierten en grandes laboratorios para los profesores que se dedican a abordarlas sin prever los resultados. La motivación que se produce en ellos por lograr avances en el dominio de estas herramientas, los mantiene conectados y los impulsa a incorporarlas.

Una de las intenciones de este artículo está soportado en el pensamiento de Fullan (2007) citado por Falco (2017), en investigar sobre las razones en que se sustenta el profesor para usar las TIC, pero no solo desde el punto de vista de su percepción, creencias, experiencias o filosofía, sino también indagando aspectos atinentes al tiempo, a las relaciones que se consolidan en ese tiempo, a la medición, cronometría, cronología y categorías de uso, en un contexto específico que es la Universidad de La Guajira. Las pretensiones de esto, también se generan desde la intención de generar información que se convierta en activo de conocimiento para la institución a fin de que use esto para la planeación de proyectos tecnológicos que estén a tono con los objetivos propuestos en dichos proyectos.

Entre los antecedentes de esta de investigación que pretende reconocer la influencia del tiempo en el uso de las TIC en clases por profesores universitarios, se relaciona un estudio sobre las creencias y conocimientos que tienen los profesores acerca de la aplicación de las TIC en la enseñanza, y cuáles son los propósitos para su uso, realizado por Caicedo y Rojas (2014). Los resultados muestran que, aunque tienen creencias positivas que podrían favorecer la apropiación de las TIC a sus prácticas educativas, solo usan de manera general herramientas de fácil dominio.

La otra investigación que se incluye es de tipo descriptivo y transversal "La aceptación de las TIC por el profesorado universitario: Conocimiento, actitud y practicidad", (Rivera-Laylle, et al., 2017) cuyo objetivo fue analizar la aceptación de las TIC como herramientas de apoyo para la docencia en universidades privadas de Guatemala, se llevó a cabo con docentes que imparten cursos de pregrado y posgrado en la modalidad escolarizada y que utilizan entornos virtuales de aprendizaje. El análisis arroja que los profesores de las universidades privadas tienen una actitud más favorable por el uso de las tecnologías de información. Y encontraron que el principal inconveniente para la integración de las TIC en el aula no es la falta de capacitación, ni la necesidad de infraestructura, ni tampoco el soporte o mantenimiento, sino más bien la resistencia de uso. 


\section{METODOLOGIA}

Para acometer la investigación se utiliza una estrategia basada en la mezcla de las metodologías cuantitativa y cualitativa. Se considera decisiva esta fusión para atender el interés de la investigación, que pretende encontrar los nexos entre el uso de las TIC en clases por profesores universitarios y la variable tiempo, en condiciones de contexto, - se refiere de manera expresa a la universidad de La Guajira-, así como la concepción de los profesores sobre el uso de TIC en clases. Una de las hipótesis que maneja esta investigación es que los profesores usan las TIC y cuentan con una postura sobre ello, y la otra, es que el tiempo influencia el uso de las TIC en clases, por ello, para explicar aspectos atinentes a esta variable y sus relaciones con la concepción y uso de TIC por profesores, se considera necesario hacer uso de una combinación de los enfoques cualitativos y cuantitativos. La metodología es ex post facto por las bondades que tiene para establecer posibles relaciones de causa - efecto observando hechos ocurridos y buscando en el pasado las causas o factores que los hayan podido ocasionar. Del enfoque cuantitativo se utiliza la estadística. Del enfoque cualitativo se usa el interpretativo. Las dimensiones asociadas a la variable tiempo que se analizan corresponden a cronometría, cronología y productividad. Se construyó un cuestionario que de manera previa fue valorada por expertos con su respectivo feedback. Fue hecha una prueba piloto con 30 profesores, que sirvió para adicionar en las frecuencias, opciones de respuesta que no habían sido tenidas en cuenta. Este cuestionario fue enviado por correo electrónico a los profesores de la Universidad de La Guajira, usando una herramienta en línea Survey monkey y a través de ella se recogieron los datos. Se usó MAXQDA para el análisis cualitativo. La encuesta se envió a 928 profesores de la Universidad de La Guajira y fue respondida por 380 superando la cota de la muestra. Se tiene en cuenta para seleccionar la muestra " $n$ " en la ecuación 1:

$$
\mathrm{n}=\mathrm{p} . \mathrm{q} /(\mathrm{e} / \mathrm{Z}) 2+\mathrm{p} \cdot \mathrm{q} / \mathrm{N}(1)
$$

Donde $p=$ margen de error, $q=$ nivel de confianza, $N=$ Universo, cantidad de profesores y $Z=$ valor crítico $=1.96, N$ : 928 docentes. Se considera un margen de error del $5 \%$ y un Nivel de confianza del $95 \% . n=0.5^{\star} 0.5 /$ $(0.05 / 1.96) 2+\left(0.5^{\star} 0.5\right) / 928 ; n=272$ profesores. (cota de la muestra)

El estudio es descriptivo correlacional porque el objetivo de la investigación es analizar la influencia de la variable tiempo en el uso de las TIC en clases y evaluar las relaciones existentes entre las variables categóricas. Las variables categóricas son la variable tiempo, con sus dimensiones, cronología, cronometría y productividad y las características de los profesores, edad, tiempo de servicio, vinculación, nivel de postgrado, facultad y programa al que pertenecen. A partir del cruce de las variables categóricas, se procedió a evaluar la relación existente entre ellas a través de la prueba de independencia chi cuadrado y de manera cualitativa, la concepción de los profesores sobre el uso de las TIC en clases y su concepción sobre cuánto tiempo usarlas en clases. El cuestionario presenta dos preguntas abiertas asociadas con esto para las que se construyeron para su análisis unas categorías. Las categorías se definieron a partir de la técnica de análisis de contenido que se realizó a los comentarios de respuesta presentados por los profesores, en los que se identificó una idea central y las respuestas asociadas a cada una de estas ideas se incorporaban a la respectiva categoría.

\section{RESULTADOS Y DISCUSIÓN}

En la Tabla 1 es posible reconocer que los profesores cuentan con una postura para usar las TIC. Mayormente ellos consideran que es altamente útil usar las TIC con un porcentaje del $59.38 \%$. El resto de los porcentajes hasta el $99.99 \%$ están repartidos entre muy útil y útil. Hay un solo profesor que expresó que no es útil. Al preguntárseles el porqué de esto, sus respuestas se organizaron haciendo uso del análisis de contenido, resultando 13 categorías. Las razones de los profesores para su postura de utilidad, están centradas mayormente en que optimiza la enseñanza, facilita el aprendizaje, sirve para el manejo de la información.

Tabla 1. ¿Cuál es su concepto de usar las TIC en clases?

\begin{tabular}{|c|c|}
\hline Opciones & Respuestas en porcentajes \\
\hline Altamente útil & $59,38 \%$ \\
\hline Muy útil & $30,21 \%$ \\
\hline Útil & $10,16 \%$ \\
\hline Poco útil & $0.00 \%$ \\
\hline No es útil & $0.26 \%$ \\
\hline
\end{tabular}

En la figura 1, se presentan las categorías sobre el sentir de los profesores frente a porqué usar las TIC en clases. En el análisis correlacional con la edad de los profesores, se encontró que ésta impacta de manera directa esta concepción que tienen los profesores con respecto a usar las TIC; para este caso, en la prueba Chi-cuadrado de independencia se obtuvo el valor de 0.021 . En todos los grupos etarios consultados, hay 
respuestas con frecuencias en lo altamente útil que es y los grupos que más lo considera son los profesores con edades entre los 31 a 40 años y los 41 a 50 años. El sexo de los docentes, impacta este tiempo de uso de TIC. Para ambos, profesores y profesoras, es importante dedicar tiempo a usar las TIC en clases.

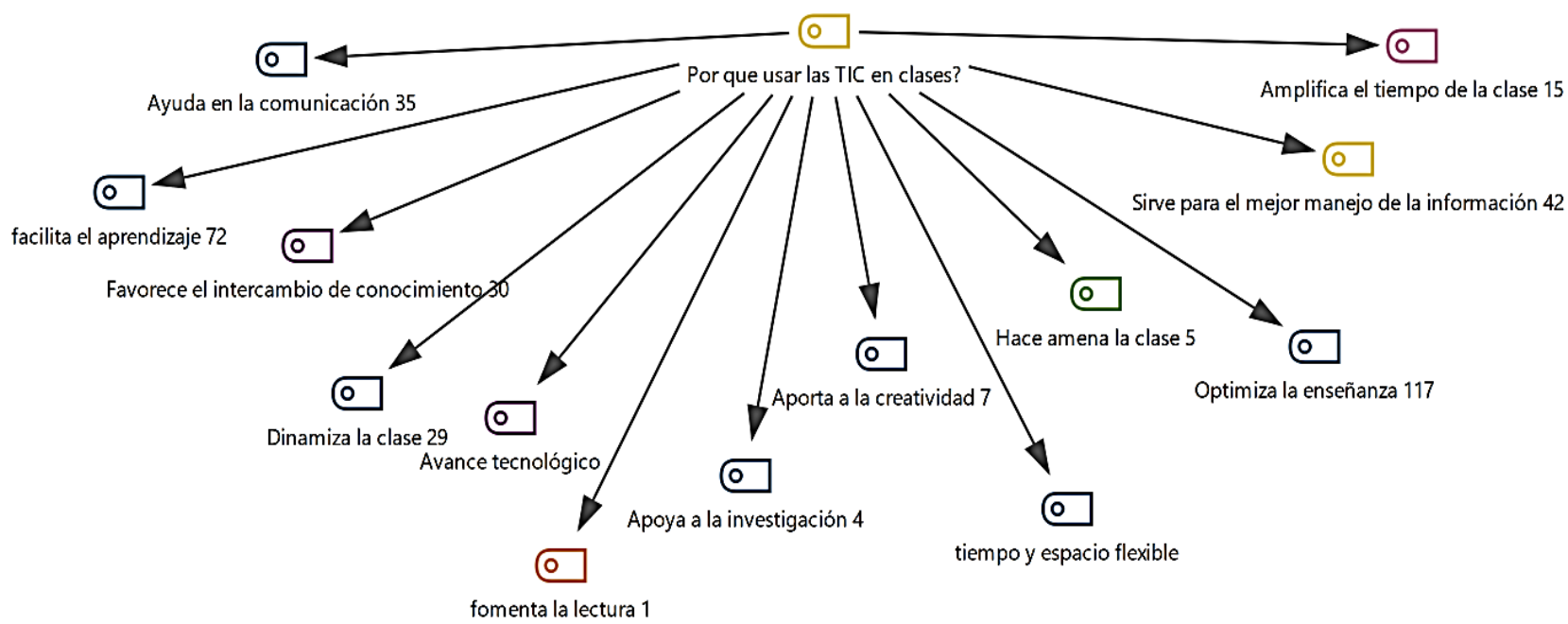

Fig. 1. Razones de la postura de los profesores ¿Por qué es útil usar las TIC en clases?

En la tabla 2 se identifica que los profesores tienen una concepción sobre cuánto tiempo usar las TIC en clases. Es valioso mencionar que un reducido grupo de profesores, (4) respondieron que no usan tiempo para TIC en clases y esto está asociado a que ellos consideran que "el profesor es el importante y el es quien debe ser el medio para enseñar todo". Estos mismos profesores en pregunta anterior indicaron que era útil usar las TIC en clases.

Tabla 2. ¿Cuánto tiempo considera que debe usar las TIC en clases?

\begin{tabular}{|c|c|}
\hline Opciones & Respuestas en porcentajes \\
\hline No necesita tiempo & $1.82 \%$ \\
\hline Poco tiempo & $3.39 \%$ \\
\hline Suficiente tiempo & $56.25 \%$ \\
\hline Mucho tiempo & $20.05 \%$ \\
\hline Todo el tiempo & $18.49 \%$ \\
\hline
\end{tabular}

En la figura 2, podemos reconocer las razones de los profesores sobre usar tiempo para las TIC en clases, que igualmente están valoradas desde pensamientos asociados al hacer pedagógico y a la eficiencia y valor del tiempo, expresados en categorías en las que hay mayormente coincidencias en que optimiza la enseñanza, debe estar en equilibrio con el tema de clases, aprendizaje autónomo y en que favorece la comunicación.

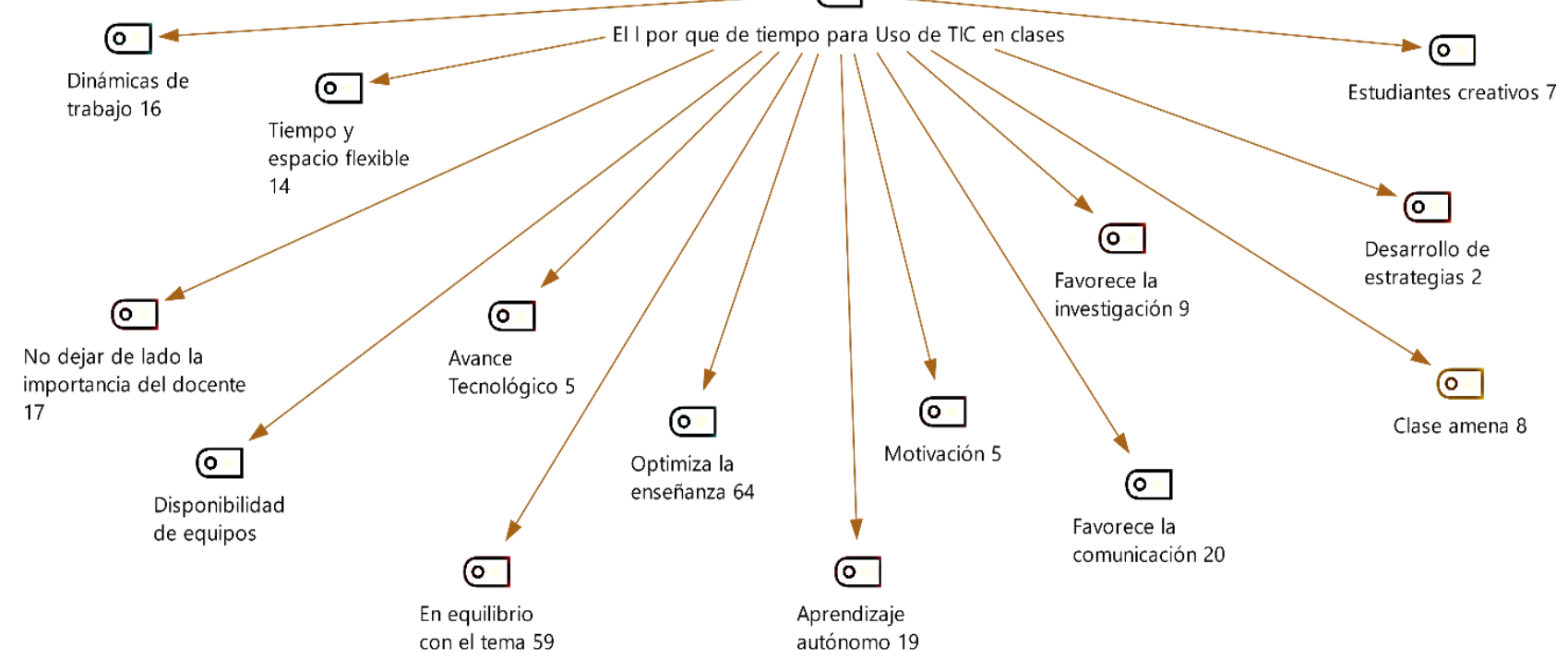

Fig. 2. Razones de los profesores sobre el tiempo para usar las TIC en clases 
En la Tabla 3 se presentan desde la cronología los resultados sobre el uso de TIC en el aula de clases en función del tiempo, a tono con lo que los profesores respondieron. Las preguntas están asociadas a franja del tiempo, uso de TIC antes de clases, durante las clases, después de clases, secuencialidad, igualdad y experiencia de uso de TIC. La tabla 4 de manera específica presentan las preguntas sobre cronometría y productividad del tiempo de uso de TIC en clases. Y la tabla 5 sobre productividad del tiempo social.

Tabla 3. Uso de TIC en el aula de clases en función del tiempo, desde la cronología.

\begin{tabular}{|c|c|c|c|}
\hline No. & Pregunta & Respuestas & \\
\hline 3 & $\begin{array}{l}\text { Franja del día en que usa las TIC } \\
\text { desde el rol del profesor }\end{array}$ & \begin{tabular}{l}
\multicolumn{1}{c}{ Franja del día } \\
Mañana \\
Tarde \\
Noche \\
Mañana y Tarde \\
Mañana y Noche \\
Tarde y Noche \\
Mañana, Tarde y Noche
\end{tabular} & $\begin{array}{c}\text { Respuestas } \\
15.84 \% \\
18.18 \% \\
9.35 \% \\
28.31 \% \\
7.01 \% \\
8.82 \% \\
18.96 \% \\
\end{array}$ \\
\hline 4 & $\begin{array}{l}\text { ¿Usa las TIC para la realización de } \\
\text { actividades antes de clases por los } \\
\text { estudiantes? }\end{array}$ & $\begin{array}{l}\text { Opciones } \\
\text { Siempre } \\
\text { Casi siempre } \\
\text { Algunas veces } \\
\text { Casi nunca } \\
\text { Nunca }\end{array}$ & $\begin{array}{c}\text { Respuestas } \\
18.44 \% \\
34.81 \% \\
37.92 \% \\
8.05 \% \\
0.78 \%\end{array}$ \\
\hline 5 & $\begin{array}{l}\text { ¿Usa las TIC para la realización de } \\
\text { actividades por los estudiantes } \\
\text { después de clases? }\end{array}$ & $\begin{array}{l}\text { Opciones } \\
\text { Siempre } \\
\text { Casi siempre } \\
\text { Algunas veces } \\
\text { Casi nunca } \\
\text { Nunca }\end{array}$ & $\begin{array}{c}\begin{array}{c}\text { Respuestas en } \\
\text { porcentajes }\end{array} \\
19.69 \% \\
43.78 \% \\
33.42 \% \\
2.33 \% \\
0.78 \% \\
\end{array}$ \\
\hline 6 & $\begin{array}{l}\text { ¿Cómo es el tiempo de uso de las TIC } \\
\text { durante las clases? }\end{array}$ & \begin{tabular}{l}
\multicolumn{1}{c}{ Opciones } \\
Secuencial \\
Con intervalos de tiempo
\end{tabular} & $\begin{array}{c}\begin{array}{c}\text { Respuestas en } \\
\text { porcentajes }\end{array} \\
25.72 \% \\
74.28 \% \\
\end{array}$ \\
\hline 7 & $\begin{array}{l}¿ E \text { El tiempo que usa las TIC es igual en } \\
\text { todas las clases? }\end{array}$ & $\begin{array}{l}\text { Opciones } \\
\text { Siempre } \\
\text { Casi siempre } \\
\text { Algunas veces } \\
\text { Casi nunca } \\
\text { Nunca } \\
\end{array}$ & $\begin{array}{c}\begin{array}{c}\text { Respuestas en } \\
\text { porcentajes }\end{array} \\
6.51 \% \\
28.13 \% \\
45.31 \% \\
15.15 \% \\
3.91 \%\end{array}$ \\
\hline 8 & $\begin{array}{l}\text { ¿El tiempo en que usan las TIC los } \\
\text { estudiantes en sus cursos, es para la } \\
\text { realización de qué tipo de } \\
\text { actividades? }\end{array}$ & \begin{tabular}{l}
\multicolumn{1}{c}{ Opciones } \\
Solo individuales \\
Solo grupales \\
Individuales y Grupales
\end{tabular} & $\begin{array}{c}\begin{array}{c}\text { Respuestas en } \\
\text { porcentajes }\end{array} \\
8.57 \% \\
13.77 \% \\
77.40 \%\end{array}$ \\
\hline 9 & $\begin{array}{l}\text { ¿La experiencia que ha tenido con } \\
\text { respecto al tiempo de uso de las TIC } \\
\text { en sus cursos, la tiene en cuenta para } \\
\text { planear las próximas actividades? }\end{array}$ & $\begin{array}{l}\text { Opciones } \\
\text { Siempre } \\
\text { Casi siempre } \\
\text { Algunas veces } \\
\text { Casi nunca } \\
\text { Nunca } \\
\end{array}$ & $\begin{array}{c}\begin{array}{c}\text { Respuestas en } \\
\text { porcentajes }\end{array} \\
24.22 \% \\
45.83 \% \\
26.30 \% \\
3.65 \% \\
0.0 \% \\
\end{array}$ \\
\hline 10 & $\begin{array}{l}\text { ¿Si se desborda o le falta tiempo } \\
\text { usando TIC en sus cursos en la } \\
\text { Universidad de la Guajira, tiene en } \\
\text { cuenta ese tiempo para la próxima } \\
\text { vez? }\end{array}$ & $\begin{array}{l}\text { Opciones } \\
\text { Siempre } \\
\text { Casi siempre } \\
\text { Algunas veces } \\
\text { Casi nunca } \\
\text { Nunca }\end{array}$ & $\begin{array}{c}\begin{array}{c}\text { Respuestas en } \\
\text { porcentajes }\end{array} \\
22.51 \% \\
37.17 \% \\
30.89 \% \\
7.07 \% \\
9.36 \% \\
\end{array}$ \\
\hline
\end{tabular}


Cronología: La franja del día en la que más usan las TIC los profesores es en la mañana y en la tarde (28.31\%). En su gran mayoría (37.92\%), los profesores Algunas veces usan las TIC para que los estudiantes realicen tareas antes de clases y casi siempre (43.78\%) les colocan actividades después de clases. El uso de las TIC en las clases es mayormente (74.28\%) en medio de otras actividades. El tiempo que usa las TIC para la mayoría de los profesores (45.31\%) algunas veces es igual en todas las sesiones de clases. Las actividades que realizan que incorporan uso de TIC, mayormente (77.40\%) son combinaciones de actividades grupales e individuales. La mayoría de los profesores $(45.83 \%)$ casi siempre tienen en cuenta el tiempo de uso de TIC de cada clase para planear las próximas actividades y si se desbordan o les falta tiempo de uso de TIC en la clase, casi siempre (37.17\%)) tienen en cuenta ese tiempo para la próxima sesión de clases. El sexo de los profesores está también relacionado con esto, de tal forma que, para ambos, profesores y profesoras en su gran mayoría, algunas veces tiene en cuenta la experiencia del tiempo de la sesión de clases usando TIC para la próxima. Su formación de postgrado también está relacionado con esto, así que los doctores, magisters, especialistas tienen en cuenta esto como experiencia para las próximas sesiones de clases. La vinculación de los profesores guarda relación con este tiempo previo y casi siempre los profesores catedráticos y de planta lo tienen en cuenta, pero los profesores contratados como Ocasionales, algunas veces lo hacen.

Cronometría: El más alto porcentaje de los profesores (33.68\%) casi siempre miden el tiempo en que usa las TIC en clases y es de una (1) hora a dos (2) horas (36.75\%) y un porcentaje un poco menor expresa que no lo tiene medido (32.55\%). La edad impacta estos resultados así que los profesores entre los 31 y 40 años y los de 51 y 60 años indican que no tienen medido el tiempo de uso de TIC, mientras que los de 41 y 50 años que son el grupo más grande de profesores, son los que las usan de manera medida entre $1 \mathrm{~h}$ y $2 \mathrm{~h}$. Los profesores $(47.27 \%)$ si usan tiempo para revisar tareas y trabajos usando TIC e indican la mayoría (33.68\%) que ese tiempo no lo tienen medido. El programa al que pertenecen los profesores está relacionado con este tiempo de revisión de trabajos así que los profesores de Administración de Empresas, Biología, Administración Marítima, Administración Turística y Hotelera, Contaduría, Economía, Negocios Internacionales, Derecho, Psicología, Licenciatura en Educación Física y Deportes, Licenciatura en Etnoeducación, Ingeniería Ambiental Ingeniería Industrial e Ingeniería de Sistemas, no lo tienen medido. Los profesores de Trabajo Social, Licenciatura en Educación Infantil e Ingeniería Civil, usan más de 1 hora. Los profesores de la Licenciatura en Música, usan de $1 \mathrm{~h}$ a $2 \mathrm{~h}$. Los profesores de Administración Pública por Ciclos propedéuticos usan de $2 \mathrm{~h}$ a 3 h y los de Ingeniería Mecánica usan de 3h a 4 h para la revisión de trabajos y tareas.

Productividad: En una selección de múltiples opciones el $72 \%$ de los profesores dedica el tiempo de uso de TIC para la enseñanza; $55 \%$ de ellos toma tiempo para la realización y revisión de tareas, así como un $50 \%$ a la comunicación con el estudiante. Dedica a la investigación un $51 \%$ y al acceso y análisis de contenidos un $49 \%$. Son múltiples las actividades que realizan los profesores durante el tiempo en el que usa las TIC en clases y entre las múltiples opciones de respuesta, se dedican al desarrollo de proyectos educativos (42.71\%), a la renovación de la docencia directa (34.48\%) y a la elaboración de planes de estudio (34.48\%). También lo hacen para resultados de investigación (32.36\%) y la escritura de artículos y libros (23.34\%). En cuanto a las interacciones comunicativas agradables entre los estudiantes asociados al tema de estudio, estas casi siempre (50.13\%) se generan durante el tiempo de uso de TIC en clases. Hay una relación entre el sexo de los profesores y esta dimensión, en tanto los profesores perciben con mayor porcentaje la generación de estas interacciones positivas que las profesoras. Se observa que existe una correlación entre la facultad a la que pertenecen los profesores y esta dimensión.

Para las interacciones comunicativas agradables entre estudiantes y profesores, se da que casi siempre $(48.49 \%)$ ocurren. Los profesores responden que casi siempre $(48.43 \%)$ se genera motivación en los estudiantes por las actividades que realizan durante el tiempo de uso de TIC. La mayoría de los profesores, más del $40 \%$, con precisión el $42.60 \%$, responde que casi nunca se generan molestias o incomodidades en sus estudiantes por las actividades que realizan durante el tiempo de uso de TIC en clases. Y en este caso, son las profesoras las que perciben mayormente que no se generan estas molestias o incomodidades en los estudiantes, que los profesores. Hay una relación entre el sexo de los profesores y esta dimensión.

Con relación a las concepciones que tienen los profesores sobre el uso de las TIC, es valioso analizar las circunstancias que se presentan en este estudio y que pueden ser objeto de discusión. Silva y Astudillo (2013) indican que la actitud que el profesor adopta respecto a las TIC favorece o es una barrera para incorporar las TIC. Los profesores en su gran mayoría evidencian que tienen una concepción positiva hacia usar las TIC en clases y esa postura se percibe en la forma como responden sobre cada una de las actividades que indican poner en marcha. Llama la atención la respuesta de cuatro (4) profesores que aunque tienen una postura positiva sobre la utilidad de las TIC en clases, cuando se les pregunta sobre sus usos, responden que no las usan. Si las concepciones son posturas verbalizadas que generan adopción de TIC, como se entiende que, habiendo una concepción positiva, luego no hay adopción. Las implicaciones que esto tiene debe llevarnos a revisar que además de partir de una actitud positiva, que es necesaria, pero no es suficiente, sería necesario 
revisar la formación tecnológica de los profesores universitarios, su eficiencia digital para analizar esta relación, concepción con adopción y con esto generar un proyecto tecnológico que pueda ser viable y efectivo.

Tabla 4. Preguntas sobre cronometría y productividad del tiempo de uso de TIC en clases

\begin{tabular}{|c|c|c|c|}
\hline$N^{o}$ & Pregunta & Respuestas & \\
\hline \multirow[t]{6}{*}{11} & \multirow{6}{*}{$\begin{array}{l}\text { ¿El tiempo que usa para las TIC en sus cursos en } \\
\text { la Universidad de La Guajira, es medido? }\end{array}$} & Franja del día & Respuestas \\
\hline & & Siempre & $25.33 \%$ \\
\hline & & Casi siempre & $33.68 \%$ \\
\hline & & Algunas veces & $26.63 \%$ \\
\hline & & Casi nunca & $9.66 \% \%$ \\
\hline & & Nunca & $4.70 \% \%$ \\
\hline \multirow[t]{7}{*}{12} & \multirow{7}{*}{$\begin{array}{l}\text { ¿Cuánto tiempo por sesiones de clases, usa } \\
\text { aproximadamente las TIC en la Universidad de La } \\
\text { Guajira, en sus cursos? }\end{array}$} & Opciones & Respuestas \\
\hline & & No lo tengo medido & $32.55 \%$ \\
\hline & & Menos de una hora & $17.32 \%$ \\
\hline & & De $1 \mathrm{~h}$ a $2 \mathrm{~h}$ & $36.75 \%$ \\
\hline & & De $2 h$ a $3 h$ & $8.66 \%$ \\
\hline & & De $3 h$ a $4 h$ & $3.67 \%$ \\
\hline & & Más de $4 \mathrm{~h}$ & $1.05 \%$ \\
\hline \multirow[t]{5}{*}{13} & \multirow{5}{*}{$\begin{array}{l}\text { ¿Toma tiempo para revisar tareas y trabajos } \\
\text { usando TIC? }\end{array}$} & Opciones & Respuestas \\
\hline & & Siempre & $34.81 \%$ \\
\hline & & Casi siempre & $47.27 \%$ \\
\hline & & Casi nunca & $16.10 . \%$ \\
\hline & & Nunca & $1.82 \%$ \\
\hline \multirow[t]{7}{*}{14} & \multirow{7}{*}{$\begin{array}{l}\text { ¿Cuánto tiempo diariamente toma para revisar } \\
\text { tareas y trabajos de los cursos que orienta en la } \\
\text { Universidad de La Guajira? }\end{array}$} & Opciones & Respuestas \\
\hline & & No lo tengo medido & $33.68 \%$ \\
\hline & & Una hora & $17.62 \%$ \\
\hline & & De $1 \mathrm{~h}$ a $2 \mathrm{~h}$ & $19.95 \%$ \\
\hline & & De $2 h$ a $3 h$ & $16.84 \%$ \\
\hline & & De 3h a 4h & $10.36 \%$ \\
\hline & & Más de $4 \mathrm{~h}$ & $1.55 \%$ \\
\hline \multirow[t]{20}{*}{15} & \multirow{20}{*}{$\begin{array}{l}\text { ¿El tiempo de uso de las TIC en sus cursos en la } \\
\text { Universidad de La Guajira, está dedicado a? }\end{array}$} & Opciones & Respuestas \\
\hline & & Enseñanza & $72.54 \%$ \\
\hline & & Investigación & $70.58 \%$ \\
\hline & & Acceso a contenidos & $46.74 \%$ \\
\hline & & Divulgación de aprendizajes & $36.79 \%$ \\
\hline & & Tareas & $55.18 \%$ \\
\hline & & Generación de pensamiento & $27.98 \%$ \\
\hline & & Análisis de contenidos & $40.41 \%$ \\
\hline & & Divulgación de información & $46.63 \%$ \\
\hline & & $\begin{array}{l}\text { Reconocimiento de } \\
\text { presaberes de los estudiantes }\end{array}$ & $23.06 \%$ \\
\hline & & Reportes de evaluaciones & $39.64 \%$ \\
\hline & & Comunicación con el & \\
\hline & & estudiante & $51.30 \%$ \\
\hline & & estudiantes & $20.98 \%$ \\
\hline & & Comunicación desde el & \\
\hline & & estudiante al docente & $36.01 \%$ \\
\hline & & Evaluaciones & $30.57 \%$ \\
\hline & & Foros Académicos & $24.09 \%$ \\
\hline & & Desarrollo institucional & $11.14 \%$ \\
\hline & & No usa las TIC & $0.78 \%$ \\
\hline \multirow[t]{10}{*}{16} & \multirow{10}{*}{$\begin{array}{l}\text { El tiempo que dedica al desarrollo de actividades en } \\
\text { las que usa TIC, en su calidad de docente, genera } \\
\text { productos académicos, tales como: }\end{array}$} & Opciones & Respuestas \\
\hline & & Proyectos educativos & $42.71 \%$ \\
\hline & & Diseño de Productos & $20.16 \%$ \\
\hline & & $\begin{array}{l}\text { Nuevos Programas } \\
\text { académicos }\end{array}$ & $9.55 \%$ \\
\hline & & Resultados de investigación & $32.36 \%$ \\
\hline & & Artículos y Libros & $23.34 \%$ \\
\hline & & $\begin{array}{l}\text { Renovación de la docencia } \\
\text { directa }\end{array}$ & $34.48 \%$ \\
\hline & & Planes de estudio & $34.48 \%$ \\
\hline & & Nuevos proyectos de & $22.28 \%$ \\
\hline & & otro & $13 \%$ \\
\hline
\end{tabular}


Tabla 5 Preguntas sobre productividad del tiempo social de uso de TIC en clases.

\begin{tabular}{|l|c|c|c|c|c|}
\hline Preguntas/ Frecuencia & Siempre & $\begin{array}{c}\text { Casi } \\
\text { siempre }\end{array}$ & $\begin{array}{c}\text { Algunas } \\
\text { veces }\end{array}$ & $\begin{array}{c}\text { Casi } \\
\text { nunca }\end{array}$ & Nunca \\
\hline $\begin{array}{l}\text { 17. ¿En el tiempo que usa las TIC en sus cursos en la } \\
\text { Universidad de La Guajira, se generan interacciones } \\
\text { comunicativas agradables entre los estudiantes asociados } \\
\text { al tema de estudio? }\end{array}$ & $35.62 \%$ & $48.49 \%$ & $13.70 \%$ & $1.37 \%$ & $0.82 \%$ \\
\hline $\begin{array}{l}18 . \text { ¿En el tiempo que usa las TIC en sus cursos en la } \\
\text { Universidad de La Guajira, se generan interacciones } \\
\text { comunicativas agradables entre usted y los estudiantes, } \\
\text { asociadas al tema de estudio? }\end{array}$ & $35.62 \%$ & $48.49 \%$ & $13.70 \%$ & $1.37 \% \%$ & $0.82 \%$ \\
\hline $\begin{array}{l}19 . \text { ¿En el tiempo que usa las TIC en la clase se genera } \\
\text { motivación en los estudiantes por las actividades que } \\
\text { realizan? }\end{array}$ & $39.01 \%$ & $48.43 \%$ & $10.99 \%$ & $1.05 \%$ & $0.52 \%$ \\
\hline $\begin{array}{l}\text { 20. ¿En el tiempo que usa las TIC en la clase se generan } \\
\text { molestias o incomodidades en los estudiantes por las } \\
\text { actividades que realizan? }\end{array}$ & $4.94 \%$ & $7.01 \%$ & $17.14 \%$ & $42.60 \%$ & $28.31 \%$ \\
\hline
\end{tabular}

\section{DISCUSION FINAL}

Hay profesores que tienen medido el tiempo, otros no y estos que aunque no lo tienen medido con reloj, de todas maneras tienen una percepción sobre la métrica del tiempo en que usan las TIC en clases, porque pueden dar una respuesta sobre el tiempo aproximado que toman para realizar actividades. Tienen activo su reloj circadiano, su reloj cronometral y su reloj cognitivo y esto les ayuda a tomar decisiones para situaciones futuras o históricas, lo que muestra desde la cronometría la influencia que el tiempo tiene en la forma como deciden usar las TIC en clases. En la quinta dimensión del tiempo (el tiempo sociopolítico) se observan aproximaciones a ella, en el sentido que los profesores expresan que usan tiempo con TIC para el desarrollo de actividades académico administrativas entre otras, pero no se midió dentro de las preguntas, si esto se hace por iniciativa propia, o porque son invitados por la institución a usar su tiempo en ello desde una planeación solicitada por la institución;

La combinación del tiempo como variable física con la forma como los profesores lo apropian motivados por su vivencia y concepción, hace que las dimensiones del tiempo influyan en lo que los profesores viven en el aula y lo contrario. Unos tienen preferencias por unos momentos de uso de TIC en la clase, para otros hay continuidad de actividades con uso de TIC dejadas de hacer en una clase con la siguiente clase y otros por ejemplo, perciben con claridad que se dan motivaciones positivas en clases entre sus estudiantes mientras usan las TIC y hay quienes tienen el uso del tiempo para TIC en clases planeado y hay quienes no. Con esto concluimos que el tiempo es una variable que influye en el uso de TIC en clases y además que lo que ocurre en las clases, influye en el uso del tiempo. Y esta última asociación, que indica que la enseñanza que ponen en marcha los profesores influye en el uso de TIC en clases, se deja para la discusión en tanto aunque estuvo en los aportes teóricos presentados en este artículo, no fue el objeto de la investigación comprobarlo ni hizo parte de sus hipótesis.

\section{CONCLUSIONES}

A partir de los resultados obtenidos, se pueden extraer las siguientes conclusiones: 1) La percepción positiva de los profesores hacia las TIC es alta y se observa que hay uso de ellas. Así como también se observa que aunque es alta la percepción positiva, hay en una mínima proporción quienes no la usan; 2) Las concepciones positivas de los profesores hacia el uso de TIC en clases, los lleva a un tiempo promedio para el uso de ellas, percibido por ellos, como "el tiempo suficiente"; 3) Fue posible identificar con precisión los usos de las TIC en función del tiempo en los que es clara una gran realización de actividades y tareas, reafirmándose que el tiempo es el elemento más importante en la estructuración del trabajo del profesor y el tiempo de uso de TIC hace parte de la estructura de la labor de enseñar; 4) Se pudieron identificar cuatro de las cinco dimensiones del tiempo, interrelacionadas entre sí, el tiempo fenomenológico, el tiempo técnico-racional, el tiempo micro político, el tiempo físico, y el tiempo sociopolítico; 5) Se confirma la apuesta que el tiempo no es independiente de lo que sucede, porque está formado por lo que se hace en el tiempo y por las relaciones entre ese tiempo y lo que se hace. Esta apuesta surge de las afirmaciones de nuestros profesores en el sentido que el tiempo que les hizo falta o les sobró lo tienen en cuenta para las próximas sesiones de clases; 6) La universalidad del tiempo afecta a los profesores en el aula. Cada uno lo hace a desiguales niveles; 7) Los profesores cuentan con una organización de la clase y de la secuencia de los tiempos que les toma hacer uso de las TIC en clases, momento a momento. Están situados en un tiempo cronológico. Tienen conciencia sobre la datación del tiempo y sobre la experiencia de cada clase, para planear la próxima; 8) La combinación del tiempo como variable física con la forma como los profesores lo apropian motivados por su vivencia y concepción, hace 
que las dimensiones del tiempo influyan en lo que los profesores viven en el aula y lo contrario; y 9) Las características de los profesores que están asociadas a tiempo, como edad, formación de postgrado, tiempo de servicio, vinculación, influyen en la forma como se usan las TIC en clases.

\section{AGRADECIMIENTOS}

Los autores agradecen a los profesores de la Universidad de La Guajira por su participación y al Centro de Investigaciones por el apoyo brindado para la publicación de los resultados.

\section{REFERENCIAS}

Adam, B., Timewatch: The social analysis of time, John Wiley y Sons, (2013)

Ancira, A. Z., y Gutiérrez, F. J. M., Integración y apropiación de las TIC en los profesores y los alumnos de educación media superior. Apertura, 3(1), 142-155, (2021)

Arancibia Herrera, M., Badia Garganté, A., Soto Caro, C. P., y Sigerson, A. L., The Impact of Secondary History Teachers' teaching conceptions on the Classroom use of Computers. Technology, Pedagogy and Education, 27(1), 101114, (2018)

Bernal-Martínez-de-Soria, A., y lbarrola-García, S., Liderazgo del profesor: Objetivo básico de la gestión educativa, (2015)

Caicedo-Tamayo, A. M., y Rojas-Ospina, T., Creencias, Conocimientos y usos de las TIC de los profesores universitarios. Educación y Educadores, 17(3), 517-533, (2014)

Cervantes-Cota, J. L., Galindo-Uribarri, S., y Smoot, G. F., The legacy of Einstein's eclipse, gravitational lensing. Universe, 6(1), 9, (2020)

Chandrasekhar, S., Newton's Principia for the common reader. Oxford University Press, (2003)

Cladellas, R., El tiempo como factor cultural y su importancia socioeconómica: Estado del arte y líneas futuras. Intangible Capital, 5(2), (2009)

Correa, A., Lupiáñez, J. y Tudela., La percepción del Tiempo: una Revisión desde la Neurociencia Cognitiva. Cognitiva, 18, 145-160, (2006)

Falco, M., Reconsiderando las prácticas educativas: TICs en el proceso de enseñanza-aprendizaje. Tendencias pedagógicas, (2017)

Ferreira, A. I., y Esteves, J.D., Perceptions of Time at Work. Personnel Review, (2016)

Gil-Madrona, P., Gómez, J. L., Aguilar-Jurado, M. Á., y Gutiérrez-Marín, E. C., Appropriate Self-Perceived behaviors in primary education pupils during sports games. Frontiers in Psychology, 11, (2020)

Ham, L., Aión, Kairós, Kronos: Una experiencia de la continuidad del tiempo. Cuadernos De Sistemática Peirceana, Número 5. (69-81), (2013)

Hjalmarsson, M., Leisure-time teacher's Reflections on Systematic Quality Work: Approaches and Challenges. Early Child Development and Care, 189(13), 2197-2205, (2019)

Hunzicker, J., From Teacher to Teacher Leader: A conceptual model. International Journal of Teacher Leadership, 8(2), 1-27, (2017)

Lafleur, C., 10 The Meaning of time: Revisiting values and aducational administration. The values of educational administration, 170. Paul Begley, Pauline Leonard. ISBN 020398322X, 9780203983225. 288, (1999)

López, M., J., Fernández L., F., Inmaculada P., S., y Garcia, O., M., The Variable "T" in Overcoming the Confrontation of Presential Vs Online Training: Mechanics of general relativity and continuing knowledge-time. In $2020 \mathrm{X}$ International Conference on Virtual Campus IEEE (JICV) 1-4, (2020)

Lucas, J., R., A treatise on time and space. Routledge, (2018)

Malquori, D., Algunas consideraciones sobre el concepto del tiempo. Pensamiento. Revista de Investigación e Información Filosófica, 67(254 S. Esp), 1007-1027, (2011)

Martinic, S., y Villalta, M., La gestión del tiempo en la sala de clases y los rendimientos escolares en escuelas con jornada completa en Chile. Perfiles educativos, 37(147), 28-49, (2015)

Morrissey, J., El uso de TIC en la enseñanza y el aprendizaje. Cuestiones y desafíos. Capítulo 7. Las TIC: del aula a la Agenda pública. Unesdoc ISBN:978-92-806-4287-2, (2007)

Oliva, M. F., Desafíos del análisis del tiempo en las ciencias sociales. INNOVA UNTREF. España, (2020)

Petkov, V., Minkowski spacetime: A hundred years later (Vol. 6). Berlin: Springer, (2010)

Rivera-Laylle, L., I., Fernández-Morales, K., Guzmán-Games, F., J., y Eduardo-Pulido, J., La aceptación de las TIC por profesorado universitario: Conocimiento, actitud y practicidad. Revista Electrónica Educare, 21(3), 99-116, (2017) 
Sáez López, J. M., Actitudes de los docentes respecto a las TIC, a partir del desarrollo de una práctica reflexiva. Escuela Abierta, ISSN: 1138-6908, (2015)

Shepherd M, Patient R., y Luzaan S., Effects of Gauteng province's educators' ICT self-efficacy on their pedagogical use of ICTS in classrooms, Heliyon, 6(4), ISSN 2405-8440, https://doi.org/10.1016/j.heliyon.2020.e03730, (2020)

Silva Quiroz, J. E., y Astudillo, A., Formación de tutores. Aspecto clave en enseñanza virtual. Revista Didasc@ lia: Didáctica y Educación, (2013)

Tammaro, R., y D'Alessio, A., Teacher training and digital competence: A pedagogical recommendation. International Journal of Digital Literacy and Digital Competence (IJDLDC), 7(2), 1-10, (2016)

Wittmann, M., y Van Wassenhove, V., The experience of time: neural mechanisms and the interplay of emotion, cognition and embodiment, (2009) 